\title{
Ambidextrie - Brauchbare Beobachtungsbrille zur aktuellen Konfliktdynamik in Organisationen?
}

\author{
Matthias Csar ${ }^{1}$ \\ Angenommen: 10. Mai 2021 / Online publiziert: 9. Juli 2021 \\ @ Der/die Autor(en) 2021
}

\section{Zusammenfassung}

Dieser Beitrag der Zeitschrift Gruppe. Interaktion. Organisation. (GIO) beschäftigt sich mit dem Konzept der organisationalen Ambidextrie. Ambidextrie (übersetzt: Beidhändigkeit) beschreibt die Fähigkeit einer Organisation auf die verschiedenen Marktdynamiken ihrer Umwelt gleichermaßen reagieren zu können. Sowohl das Kerngeschäft wird kontinuierlich optimiert als auch die Entwicklung von Innovation vorangetrieben. Nach der theoretischen Einbettung des Ambidextriebegriffs wird auf ein Fallbeispiel eingegangen und die Theorie auf ihre Praktikabilität hin reflektiert. Es wird der Annahme nachgegangen, dass es sich bei dem Ambidextriebegriff um ein aktuelles Erklärungsmodell handelt, das die Konfliktdynamik zwischen den Marktanforderungen von außen und der operativen Abwicklung im Inneren der Organisation in ein aktuelles Gewand kleidet.

Schlüsselwörter Ambidextrie · Exploration und Exploitation · Konfliktdynamik · Veränderungsmanagement · Organisationsentwicklung $\cdot$ Digitale Transformation $\cdot$ Organisationales Lernen

\section{Ambidexterity-A useful perception concept for current conflict dynamics in organizations?}

\begin{abstract}
The article deals with the concept of organizational ambidexterity. Ambidexterity describes the ability of an organization to react equally to the different market dynamics of its environment. As well existing business is secured by the continuous increase in efficiency as future trends are recognized and shaped by own innovation activities. After the theoretical embedding of the concept, a case study is reflected. The assumption is followed that the concept of ambidexterity is a current explanatory model that puts the conflict dynamics between external market requirements and operational processes inside the organization into a modern guise.
\end{abstract}

Keywords Ambidexterity · Exploration and Exploitation · Conflict Dynamics · Change management · Organization development · Digital transformation · Organizational learning

\section{Organisationale Ambidextrie - Theoretische Konzeptionen}

In der Diskussion zu den neueren Organisationstheorien erlangt der Begriff der organisationalen Ambidextrie zunehmend Aufmerksamkeit (vgl. hierzu eine Auflistung relevan-

Matthias Csar

office@matthiascsar.com

1 FH Salzburg - University of Applied Sciences, Salzburg, Österreich ter Artikel im Forschungsfeld der Ambidextrie bei Birkinshaw und Gupta 2013, S. 289 sowie Fojcik 2015, S. 37 ff.). Ambidextrie, übersetzt Beidhändigkeit ${ }^{1}$, beschreibt die Fähigkeit einer Organisation auf die verschiedenen Marktdynamiken ihrer Umwelt gleichermaßen reagieren zu können. Auf der einen Seite wird das bestehende Geschäft durch kontinuierliche Effizienzsteigerung gesichert. Auf der an-

\footnotetext{
${ }^{1}$ Ambidexterität (aus dem lateinischen ambo „beide“ und dextera „rechte Hand") beschreibt die Fähigkeit sowohl die rechte als auch die linke Hand gleichermaßen benutzen zu können.
} 
Tab. 1 Merkmale idealtypischer Exploit- und Explore-Ausprägung in Organisationen

\begin{tabular}{|c|c|c|}
\hline Merkmal & Exploit & Explore \\
\hline Strategische Zielsetzung & $\begin{array}{l}\text { Kurzfristiger Planungshorizont, Ausschöpfen bestehen- } \\
\text { der Rendite, Vorantreiben inkrementeller Innovation }\end{array}$ & $\begin{array}{l}\text { Langfristiger Planungshorizont, Suche nach neuen } \\
\text { Renditequellen, Vorantreiben radikaler Innovation }\end{array}$ \\
\hline Struktur & Zentralisiert, mechanistisch, funktional geschlossen & Dezentralisiert, organisch, projektorientiert offen \\
\hline Prozesse & $\begin{array}{l}\text { Orientierung und Optimierung bestehender Prozessab- } \\
\text { läufe, Kosten- \& Effizienzorientierung }\end{array}$ & $\begin{array}{l}\text { Flexibilitätsorientierung, Abkehr von Linienvorgaben, } \\
\text { Systematisches Experimentieren mit Unbekannten }\end{array}$ \\
\hline Steuerung \& Kontrolle & $\begin{array}{l}\text { Detaillierte Planungs- \& Budgetvorgaben, Verfahrens- \& } \\
\text { Ergebnissteuerung, laufende Überprüfung }\end{array}$ & $\begin{array}{l}\text { Offene Planungs- \& Budgetvorgaben, Vertrauen in } \\
\text { Selbststeuerung, seltene Überprüfung }\end{array}$ \\
\hline Anreizsysteme & $\begin{array}{l}\text { Performance fokussiert auf die individuelle Leistung des } \\
\text { Einzelnen/des Teams }\end{array}$ & $\begin{array}{l}\text { Performance fokussiert auf die Innovationskraft des } \\
\text { gesamten Unternehmens }\end{array}$ \\
\hline Führung & Top down, autoritär, aufgabenorientiert & Partizipativ, visionär, beziehungsorientiert \\
\hline $\begin{array}{l}\text { Fähigkeiten \& Lernen } \\
\text { der MitarbeiterInnen }\end{array}$ & $\begin{array}{l}\text { Verbesserung von vorhandenen Ressourcen und Kompe- } \\
\text { tenzen (Fokus auf Beschaffung - Produktion - Marke- } \\
\text { ting) }\end{array}$ & $\begin{array}{l}\text { Entwicklung neuer Ressourcen und Kompetenzen } \\
\text { (Erweiterung um unternehmerisches Denken) }\end{array}$ \\
\hline
\end{tabular}

deren Seite werden zukünftige Trends am Markt erkannt und durch eigene Innovationsaktivtäten mitgestaltet.

Diese Fähigkeit einer Organisation, zwei unterschiedliche Aktivitäten in Reaktion auf ihre Umwelt gleichermaßen abwickeln zu können, ist in der kontinuierlichen Entwicklung im Spannungsfeld zwischen Stabilität und Wandel nicht gänzlich neu. Zu Beginn der 1990er Jahre beschrieb James March (1991) im Kontext der Innovationsforschung zum ersten Mal das Spannungsfeld zwischen exploitativem und explorativem Lernen einer Organisation (ebd. S. 72). Dabei stellt sich für Unternehmen die Frage, wie es gelingen kann, sowohl das laufende Kerngeschäft zu sichern (Exploit) als auch aktiv Neuerungen und Innovationen im eigenen Produktangebot hervorzubringen (Explore). „Exploration includes things captured by terms such as search, variation, risk tasking, experimentation, play, flexibility, discovery, innovation. Exploitation includes such things as refinement, choice, production, efficiency, selection, implementation, execution“, so March (ebd. S. 1).

Duncan (1976) wiederum erwähnte als Erster den Begriff der organisationalen Ambidextrie. Dieser wurde von Tushmann und O'Reilly 1996 übernommen und zum ersten Mal als zentrale Kompetenz zukunftsfähiger Organisation beschrieben, indem es ihr gelingt zwei strategische Ausrichtungen gleichermaßen zu verfolgen (vgl. Tushmann und O'Reilly 1996, S. 24). Auch hier lag der Fokus auf der Produktentwicklung und dem Hervorbringen von Innovation, in dem Fall inkrementeller (exploitatives Vorgehen) gegenüber radikaler bzw. diskontinuierlicher Innovation (exploratives Vorgehen). Organisationen sollten beginnen, in der von einem Effizienzstreben geprägten Wettbewerbsdynamik eine langfristigere Perspektive einzunehmen, die sich um die Entwicklung zukünftiger Produktangebote sorgt und die das bestehende Geschäft im Zweifel obsolet macht. „The ability to simultaneously pursue both incremental and discontinuous innovation and change that result from hos- ting multiple contradictory structures, processes, and cultures within the same firm" (ebd. S. 24).

Der Begriff der Ambidextrie beschreibt die Gleichzeitigkeit im organisationalen Alltag, in dem auf der einen Seite die Stabilisierung und Optimierung bestehender Prozesse und Strukturen einer Organisation betont werden und auf der anderen Seite ein kontinuierliches Suchen, Experimentieren und Verändern der Produktstrategien als Notwendigkeit zur Zukunftssicherung des eigenen Geschäfts hervorgehoben werden.

Tab. 1 (in Anlehnung an Fojcik 2015, S. 54-55; March 1991, S. 72 ff.; Tushman und O'Reilly 2004, S. 80) gibt einen Einblick über die idealtypischen Ausprägungen der beiden Pole Exploit und Explore. Verschiedene Merkmale von Organisation lassen sich der spezifischen Logik nach beispielhaft unterscheiden.

Die beiden Modalitäten unterscheiden sich insofern nicht nur in den spezifischen strategischen Ausrichtungen, sondern resultieren aufgrund unterschiedlicher Erwartungshaltungen an Führungskräfte und Mitarbeiter*innen in zwei konträren Unternehmenslogiken (vgl. aktuell dazu auch Exner et al. 2020, S. 66). Diese erscheinen auf den ersten Blick als unvereinbar und inkompatibel. Spannungen und Widersprüche sind inhärent und zeigen sich im strukturellen Design, in der organisatorischen Steuerung oder in der Unternehmenskultur. Laut March (vgl. 1991, S. 72) konkurrieren Exploitation und Exploration immer auch um knappe Ressourcen, wodurch das Management permanent angehalten ist, zwischen kurzfristiger Produktivität und langfristiger Innovation $\mathrm{zu}$ entscheiden.

Gleichzeitig finden sich die beiden Ambidextrie-Dimensionen in einer paradoxen Verknüpfung wieder, in der sie sich sowohl gegenseitig bedingen als auch wechselseitig ausschließen (vgl. Lavie et al. 2010). Stehen die spezifischen Merkmale der vollen Ausprägung nach im krassen Widerspruch zueinander, so sind Exploitation und Exploration zugleich aufeinander angewiesen. Exploitation schafft 
die Ressourcenbasis für Exploration, sie finanziert sozusagen die Freiheiten für Neuentwicklung und Innovation in der Gegenwart. In der Gegenrichtung entwickelt ein exploratives Vorgehen neues Wissen und Kompetenz, welche wiederum Voraussetzung für ein späteres exploitatives Vorgehen und damit den Markterfolg in der Zukunft sichert. Diese Paradoxie stellt für Organisationen eine große Herausforderung dar, da immer aufs Neue zu entscheiden ist, wie viel Gegenwart zugunsten der Zukunft aufgegeben werden muss (vgl. ebd. S. 117 ff.).

In der Literatur werden drei Möglichkeiten beschrieben, wie Unternehmen mit dem Paradoxon einer ambidextren Organisation umgehen können. Nach Tushman und O'Reilly (2013, S. $328 \mathrm{ff}$.) wird zwischen der sequenziellen, der strukturellen und der kontextuellen Ambidextrie unterschieden. Sowohl bei der sequenziellen als auch der strukturellen Ambidextrie geht man davon aus, dass Exploit- und Explore-Aktivitäten nicht gleichzeitig in einer Organisationseinheit, sondern getrennt voneinander umgesetzt werden müssen. Sequenziell, indem man exploitatives von explorativem Vorgehen zeitlich trennt. Eine gewisse Zeitperiode lang wird einmal die Effizienz und einmal die Innovation betont und befördert. Strukturell, indem beide Aktivitätsmodi sowohl operativ wie räumlich getrennt werden. Ein paralleles Vorantreiben beider Geschäftsaktivitäten kann so sichergestellt werden. Beide Ambidextrieformen versuchen die Widersprüche divergierender Organisationslogiken durch zeitliche bzw. strukturelle/räumliche Trennung zu lösen. Dabei wird der Widerspruch jedoch lediglich auf die übergeordnete Organisationsebene geschoben und das obere Management gefordert, das Spannungsfeld zwischen Exploit und Explore zu balancieren.

Die dritte Form wird in der Literatur als kontextuelle Ambidextrie (vgl. Gibson und Birkinshaw 2004) bezeichnet. Dabei wird auf zeitliche bzw. strukturelle Lösungspuffer verzichtet und davon ausgegangen, dass organisationale Ambidextrie vom gesamten Unternehmen behandelt werden muss. Sowohl Führungskräfte als auch Mitarbeiter*innen werden angehalten sich für exploitatives und exploratives Vorgehen gleichermaßen zu engagieren und ein gemeinsames Verständnis für die Notwendigkeit beider Aktivitäten zur Sicherung der Unternehmenszukunft zu entwickeln. Kontextuelle Ambidextrie stützt die Annahme eines „sowohl als auch“ Denkens, indem sich Kreativität und Flexibilität einerseits sowie Qualität und Effizienz anderseits nicht zwangsläufig ausschließen. Das Management spielt dabei eine wesentliche Rolle, indem es einen Kontext schaffen muss, der durch entsprechende Anreize sowohl Konvergenz (Alignment) als auch Divergenz (Adaptability) unterstützt (vgl. ebd. S. 210). Hier lässt sich bereits annehmen, dass ein Ausbalancieren dieser Spannungspole Quelle von persönlichem Stress und Konflikt auf Rücken der handelnden Führungskräfte ist.
Auch wenn Tushman aktuell von einer Bevorzugung struktureller Ambidextrie in der Veränderung von Organisation spricht, „man kann neue Triebe nur hochpäppeln, wenn man sie vom ausgewachsenen Baum trennt" (im Interview mit Schumacher 2020, S. 9), stellt im Diskurs von Fach- und Berater*innencommunitys die Entwicklung kontextueller Ambidextrie den Idealmodus im Umgang mit den widersprüchlichen Exploit- und Explore-Anforderungen in Unternehmen dar (vgl. etwa Duwe 2018 oder Schumacher und Wimmer 2018). Zu welchen Herausforderungen es in der Praxis kommt und wie praktikabel das Ambidextriekonzept ist, wird in Folge mit der Reflexion eines Fallbeispiels diskutiert.

\section{Organisationale Ambidextrie - Konfliktfelder in der Praxis}

Ein IT-Unternehmen hat die letzten Jahre Schritte gesetzt, um den Herausforderungen der digitalen Transformation offensiv zu begegnen. Nach drei Jahren struktureller Trennung wird die ausgegliederte Innovationseinheit in der Softwareentwicklung zurückgeführt und mit den anderen Entwicklerteams verbunden. Die Unverbindlichkeit und Freiheiten explorativen Vorgehens treffen dabei auf historisch und hierarchisch gewachsene Prozesse der Effizienzsteigerung. ${ }^{2}$

Das IT-Unternehmen traf vor drei Jahren die Entscheidung für ein zukunftsträchtiges Produktsegment eine neue Businessunit zu gründen. Ein 8-köpfiges Team (Produktmanager und Softwareentwickler) wurde strukturell von der Linie und der Abwicklung des bestehenden Geschäfts entkoppelt und bekam die Freiheit, sich voll und ganz der Forschung und Entwicklung des neuen Produkts zu widmen. Die Explore-Einheit war klein, dezentral organisiert und unterschied sich von Beginn weg in der Arbeitsweise im Team. Nicht so wie im weitaus größeren Softwarebereich (Exploit-Einheit mit ca. 60 Entwicklern) war man auf standardisierte Prozesse und Kontinuität bemüht, sondern konnte sich frei dem Erlernen neuer technischer Möglichkeiten und Trends am Markt widmen. Das Unternehmen entschied sich drei Jahre lang für den Weg der strukturellen Ambidextrie, räumlich und operativ voneinander getrennt, auf dem das Top Management angehalten war die Balance zwischen Exploitation und Exploration zu halten. Diese Aufgabe erwies sich über die Zeit als äußerst an-

\footnotetext{
${ }^{2}$ Die Auszüge aus dem Fallbeispiel stammen aus eigenen Beobachtungen und Gesprächen mit den Beteiligten. Das IT-Unternehmen wurde von mir in verschiedenen Coaching-Zusammenhängen begleitet. Der Transformationsprozess und die Reintegration der Innovationseinheit wurde von dem Unternehmen über einen intern aufgesetzten Organisationsentwicklungsprozess gestaltet.
} 
spruchsvoll, da ein Auseinanderdriften der Arbeitskulturen zunahm, Neid und Missgunst aufgrund als ungleich wahrgenommener Ressourcenverteilung stiegen und inhaltliche Schnittstellen zwischen den Produkten immer schwerer zu bearbeiten waren. Für das Management stellte sich das Problem der Integration der eigenen Wertschöpfungskette, da heterogene Einheiten auf homogene, übergeordnete Ziele ausgerichtet werden mussten (vgl. dazu auch Tushman und O'Reilly 2013, S. 328).

Nach drei Jahren Versuch, strukturelle Ambidextrie zu bewältigen, entschied man sich für den Change und die Reintegration der Innovationseinheit in den Softwarebereich. Das Entwicklerteam der „neuen Welt“ sollte sein Wissen an die anderen Teams weitergeben und sich der Linienorganisation und somit der hierarchischen Steuerung wieder anschließen. Das Produktmanagement sollte beginnen, bestehende Kundenwünsche mit visionären Angeboten zu verbinden und divergierende Vorstellungen selbständig zu erkennen und zu lösen. Die Erwartungshaltung des Topmanagements für den Change war eindeutig, die Entwicklung einer kontextuellen Ambidextrie jedoch herausfordernd. Folgende Konfliktfelder waren zu beobachten:

- Unsicherheit in Strategie und Vision - Kalkulation versus Einschätzung

- Unsicherheit in der Herangehensweise - Routine versus Experiment

- Unsicherheit in der Führung - Kontrolle versus Freiheit

\subsection{Unsicherheit in Strategie und Vision - Kalkulation versus Einschätzung}

Ein erstes Konfliktfeld in der Zusammenführung der beiden Unternehmenseinheiten war die Auslegung der Zukunft. Von Beginn weg war das Topmanagement um Klarheit bemüht, welche strategischen Ziele kurz-, mittel- und langfristig gelten sollten. Die Vision sollte ein positives Bild einer neuen Produktwelt vermitteln, in der die innovativen Aktivtäten der letzten Jahre einen zentralen Platz fänden. Die Bemühungen des Topmanagements wurden jedoch durch konsequentes Hinterfragen von Mitarbeiter*innenseite irritiert. Zu unklar war der Rahmen, wie viel Freiheit den Explore-Aktivitäten zur weiteren Entwicklung von Innovation zugestanden bzw. wieviel Anpassung an Exploit-Tätigkeiten notwendig war.

Es kann angenommen werden, dass hinter dieser Skepsis das Ambidextrieproblem der Ressourcenverteilung steckt (vgl. March 1991, S. 72). Für die Mitarbeiter*innen war nicht klar, ob mit der Zusammenführung stärker in die Optimierung des bisher erlangten Wissens unter Nutzung der bestehenden Prozesse investiert wird oder ob man den Explore-Aktivitäten weitere Zugeständnisse und Freiheiten einräumen werde. Die Wahrnehmung von Ressourcenknapp- heit wirkte stärker als das kommunizierte Bild von Vision und Zukunft, da dem Management immer eine „EntwederOder-Entscheidung" zwischen genauer Kalkulation versus vager Einschätzung der Marktentwicklung zugeschrieben wurde.

Ganz im Sinne von: „Die wissen schon wo's hin geht, der Markt entwickelt sich so“ "gegenüber dem Zweifel „Ob das wirklich die richtige Entscheidung war, setzen wir auf das richtige Pferd?" Der Wunsch der Mitarbeiter*innen nach Orientierung und unternehmerischer Zielgenauigkeit ging im strategischen Spannungsfeld zwischen Skalierbarkeit gegenüber Unverbindlichkeit unter. Dem wirtschaftlichen Erfolg bestehender Produkte wird die fehlende Gewissheit über die Gewinnchancen möglicher Innovationen gegenübergestellt.

\subsection{Unsicherheit in der Herangehensweise - Routine versus Experiment}

In der Zusammenführung der beiden Unternehmenseinheiten wurden kulturelle Gräben sichtbar, die sich durch die strukturelle Trennung von Exploit- und Explore-Aktivitäten in den letzten drei Jahren aufgebaut hatten. Der Konflikttheorie nach nicht verwunderlich (vgl. Stufenmodell bei Glasl 2013), wurde die jeweils andere Partei abgewertet, deren Leistung für die Zukunft des Unternehmens geschmälert sowie die Arbeitsweisen des jeweils anderen als konservativ-langsam bzw. als abgehoben-idealistisch bezeichnet (siehe auch die Konfliktarten bei Schwarz 2010, S. $193 \mathrm{f}$. sowie die Diskussion zu klassischem versus agilem Projektmanagement in der Softwareentwicklung, etwa Kuster et al. 2018 oder Timinger 2017).

In den Zuschreibungen der Mitarbeiter*innen war die jeweils andere Unternehmenseinheit am „falschen Dampfer“. Für die Exploit-Vertreter*innen, die in der Mehrzahl waren, jahrzehntelange Unternehmensgeschichte im Rücken hatten und für $99 \%$ des gegenwärtigen Umsatzes verantwortlich waren, schienen die Explore-Aktivitäten als Geldverschwendung. Der Produktmanager der Innovationseinheit wurde als arrogant, besserwisserisch und weltfremd beschrieben, die Softwareentwickler als Agilitätsideologen abgetan. Die Explore-Gruppe wurde durch diese Zuschreibungen wiederum in ihrem Bild bestätigt, dass die strukturelle Trennung der letzten drei Jahre richtig war. Unter den bestehenden Strukturen und mit den beteiligten Personen war eine gemeinsame Linie kaum zu finden. $\mathrm{Zu}$ unterschiedlich waren die Arbeitsweisen und das Verständnis von Softwareentwicklung, zu verschieden die Zielvorstellungen, was die Produktenwicklung betraf.

Diese Beobachtung zeigte, dass sich in den letzten drei Jahren der Trennung die beiden Dimensionen Exploitation und Exploration zu zwei unterschiedlichen Kulturen entwickelt hatten. Im Veränderungsprozess und der Zusam- 
menführung der beiden Aktivitätsmodi zeigten sich zwei unterschiedliche Verhaltenslogiken, die spürbar in Konflikt standen. Benötigt es für das Erhalten von Exploit-Tätigkeiten Disziplin, Planung und Fokus, sind für die ExploreAktivitäten Zeit, Offenheit und auch Zufall gefragt. In der Entwicklung kontextueller Ambidextrie trifft der Wunsch nach Erhalt der Routine auf den Bedarf des Experiments. Die Zusammenarbeit ist geprägt von der Auseinandersetzung , so wie immer, denn das hat sich bewährt“ gegenüber „,bitte anders, weil sonst kommen wir zu keinen neuen Ideen!"

In der Literatur zur Ambidextrie kann hierzu die Verbindung zur „Failure bzw. Success Trap“ gezogen werden. Levinthal und March (1993) sehen innerhalb der Exploit-Explore Dynamik zwei Verdrängungseffekte, die in Unternehmen auftreten können, wenn die Vertreter*innen der jeweiligen Dimension in Konkurrenz um die Wichtigkeit ihres Daseins bzw. in Konkurrenz um Ressourcen treten. Auf der einen Seite droht die „Failure Trap“ (ebd. S. 105), die Misserfolgsfalle, die dann entsteht, wenn die explorative Suche nach Innovation, die vom Experimentieren lebt und Fehleinschätzungen legitimiert, ein wiederholtes Scheitern mit sich bringt. Anstatt innezuhalten, ExploitMeinungen wie Effizienzkritik zu zulassen, wird weiterhin auf einer explorativen Haltung beharrt. Die Misserfolgsfalle entsteht, wenn auf die Veränderungen am Markt kontinuierlich versucht wird, mit Innovation zu antworten, jedoch Irrtümer permanent produziert werden und jede Innovation einen Misserfolg nach dem anderen hervorruft. Wiederholtes Scheitern im unreflektierten Wunsch nach Exploration wird wahrscheinlicher als die Rückbesinnung auf Exploitation-Tätigkeiten.

Auf der anderen Seite kann von einer „Success Trap“ (ebd. S. 106), der Erfolgsfalle, gesprochen werden, wenn der gegenwärtige wirtschaftliche Erfolg von Exploit-Tätigkeiten konsequent hervorgehoben und kein Bedarf darin gesehen wird, in Exploration zu investieren. Sieht eine Organisation konstant mehr Anreiz zur Wiederholung als zur Veränderung erfolgsversprechender Perspektiven, Abläufen und Entscheidungen, so läuft sie Gefahr der Erfolgsfalle zu erliegen, wenn sich die Marktanforderungen und Kundenwünsche ändern. Dabei tragen Routinen, die in der Vergangenheit erfolgreich waren, zur Festigung nächster Entscheidungen bei, die wiederum keine Kapazitäten für Exploration-Tätigkeiten freisetzen.

Im Fallbeispiel war die Bereitschaft, sich auf die andere Dimension einzulassen, gering. Dem Festhalten an Routinen stand der Wunsch nach Ausprobieren und Experimentieren gegenüber. Es wirkte so, als würde man der jeweils anderen Partei zuschreiben, sich bereits in einer der beiden Fallen zu befinden: Die Innovationseinheit wurde von Exploit-Vertreter*innen mit dem Risiko verbunden, zu viele unterentwickelte Ideen mit zu wenig verwertbarem Output zu generieren. Den Exploit-Vertreter*innen wurde wiederum nachgesagt, den kurzfristigen Erfolg vor Augen zu haben und damit die langfristige Anpassungsfähigkeit zu opfern.

\subsection{Unsicherheit in der Führung - Kontrolle versus Freiheit}

Ein drittes Konfliktfeld entwickelte sich aus der Unsicherheit heraus, welches Führungsverständnis in Zukunft gelten sollte. Neben der generellen Neudefinition von Zusammenarbeit zwischen den Teams, war vor allem die Führung angehalten, sich durch die auftretenden Widersprüchlichkeiten zu manövrieren. Um die Integration des Exploration-Teams nicht von Beginn an zu gefährden, war der Bereichsleiter der Softwareabteilung gefordert, sein über Jahre aufgebautes Verständnis von richtiger Führung zu hinterfragen. Die Zusammenarbeit mit dem technischen Teamleiter der Innovationseinheit entpuppte sich dabei als äußerst schwierig, da dieser, mit der Erfahrung der vergangenen drei Jahre, einen ganz anderen Führungskontakt zu seinen Teammitgliedern pflegte. Diese waren es die Jahre über gewohnt, Dinge explizit anders zu machen, bisherige Lösungswege in Frage zu stellen und selbst strategische Entscheidungen in der Produktenwicklung zu treffen. Dem Bereichsleiter der Softwareentwicklung war wiederum das Einhalten aufgestellter Prozesse und Leitlinien wichtig, die für Transparenz und Vergleichbarkeit zwischen den verschiedenen Teams im Softwarebereich sorgten. Einem öffnenden Führungsverhalten in der kleinen Innovationseinheit stand ein schließendes Führungsverhalten in der weitaus größeren Softwareabteilung gegenüber. Die eine Führungskraft forderte ihre Mitarbeiter*innen auf Ideen einzubringen, die andere wiederum erwartete sich Ideen umzusetzen.

In der Literatur zu kontextueller Ambidextrie finden sich genügend Modelle, die diese Beobachtung und das Spannungsfeld, in dem sich Führung permanent befindet, stützen. Exner et al. (2020) sprechen von der Fähigkeit zur dualen Steuerung, nämlich ,zwei pragmatisch verschiedene, notwendige Eigenlogiken - im Sinne der Lebensfähigkeit des ganzen Unternehmens - im Unternehmen zu differenzieren, bewusst $\mathrm{zu}$ gestalten und damit wirksam $\mathrm{zu}$ steuern“ (ebd. S. 64).

Expliziter hebt Duwe (2018) die unterschiedlichen Arten der Führungskommunikation hervor, die im Aufbau von kontextueller Ambidextrie reflektiert und ggf. im Spannungsfeld zwischen Exploitation und Exploration vereinheitlich werden muss. Das Wesen der Exploit-Kommunikation steht für Klarheit im „Senden von Information. Formalisiert, organisiert, zentralisiert, offiziell, hierarchiezentriert“" (ebd. S. 52). Dem gegenüber steht ein Explore-Kommunikationsstil, der „Realität durch Interaktion“ erkennt 
und der sich „eher informell, weniger straff und dezentralisiert organisiert" (ebd. S. 52).

Wie im Fallbeispiel erlebt, erfordert die Entwicklung kontextueller Ambidextrie Führung auf paradoxe Weise heraus. Auf der einen Seite muss an eigenen Prinzipien und dem erprobten Führungsverständnis festgehalten werden, weil sonst Erfolgsfaktoren bisheriger Zusammenarbeit zerstört werden. Auf der anderen Seite muss Führung die explorativen Erwartungshaltungen von Mitarbeiter*innen nach mehr Selbstständigkeit und Flexibilität akzeptieren und steuern lernen. Führung befindet sich in einem paradoxen Erwartungsfeld zwischen Kontrolle und Freiheit.

Szlang und Bruch machen in ihrer Studie (2020) dazu deutlich, dass ein bewusster Führungsumgang mit dem Widerspruchsfeld zwischen Exploitation und Exploration positiven Einfluss auf die Unternehmensleistung hat. Sie beschreiben den Aufbau eines ,ambidextren Führungsklimas“ (vgl. ebd. S. 189) als wichtige Organisationsleistung in Unternehmen, die sich mit zunehmenden Wettbewerbsdruck konfrontiert sehen. Von einem ambidextren Führungsklima kann dann die Rede sein, wenn Führungskräfte einen ähnlichen Führungsstil im Umgang mit den widersprüchlichen Herausforderungen zeigen. Mitarbeiter*innen nehmen diesbezüglich eine Konstanz und Einheitlichkeit in der Führungsmannschaft wahr. Aktion und Reaktion emergiert laut Szlang und Bruch in der Führungskultur eines Unternehmens: „Führungskräfte müssen aktuelle und neue Aktivitäten in Einklang bringen, kurz- und langfristiges Denken kombinieren, eine emotional ansprechende Vision entwickeln und dabei gleichzeitig auf Umsetzung fokussiert sein“" (ebd. S. 188).

Im Fallbeispiel wurde dieses Bild von Führung vom Top Management an die betroffenen Führungskräfte durchaus kommuniziert. Jedoch wurden der Entwicklung und Handhabe von Führung im Rahmen zweier diametralen Erwartungshaltungen wenig Zeit, Raum und Ressourcen zur Verfügung gestellt. Der Aufbau eines ambidextren Führungsklimas klingt in der Theorie gut, scheint in der Praxis aber umso schwieriger umsetzbar zu sein.

In der Reflexion dieses Fallbeispiels kann der Eindruck entstehen, dass sich die erforderlichen Veränderungen im bestehenden Geschäft (Strategie, Prozess, Führungsbild, etc.) sowie die Wünsche nach loseren Prozessen zur Entwicklung von Neuem mehr blockieren als unterstützen. Es werden in der Literatur kaum sichtbare Praxisbeispiele sowie belastbare Hinweise der Forschung gefunden, die eine erfolgreiche Verzahnung und Wechselwirkung von Exploitation und Exploration innerhalb unternehmerischer Zukunftssicherung dokumentieren (vgl. aktuell den Versuch von Gergs und Lakeit 2020, S. $107 \mathrm{ff}$. verschiedene Fallbeispiele zur Umsetzung von Ambidextrie in Unternehmen zu skizzieren und die Brücke zwischen Theorie und Praxis zu schlagen).
Es entsteht der Eindruck, dass das Konzept der Ambidextrie mehr Theoriegerüst als Anwendungskonzept ist. Gleichzeitig stellt es ein willkommenes Beschreibungsmodell von Konfliktdynamiken in Zeiten des digitalen Wandels dar, das momentan in Berater*innenkreisen und der Managementliteratur en vogue ist.

\section{Organisationale Ambidextrie - Konfliktdynamik in neuem Gewand}

Den Ratschlägen zum Aufbau einer ambidextren Organisation und zur Entwicklung eines ambidextren Führungsverständnisses wird momentan viel Aufmerksamkeit gewidmet. Sowohl im aktuellen Managementdiskurs (vgl. etwa die Forschungsstudie des Hernstein Instituts 2020) als auch in diversen Ratgebern zum Thema Führung und Organisationsentwicklung (etwa Duwe 2018; Schneeberger und Habegger 2020 oder Gergs und Lakeit 2020) wird Ambidextrie als die zentrale Herausforderung für Unternehmen der $\mathrm{Zu}$ kunft betrachtet. Dabei stellt sich allerdings die Frage, was an dem seit den 1990er Jahren wissenschaftlich elaborierten Konzept zum Spannungsfeld zwischen Exploitation und Exploration im Diskurs der Organisationsentwicklung heutzutage als relevant und ,neu“ bezeichnet werden kann. So gesehen könnte der Ambidextriebegriff mehr sprachliche Verkleidung altbekannter Spannungsfelder und Paradoxien in Organisationen darstellen.

\subsection{Was für die Beschreibung ambidextrer Dynamiken in Organisationen spricht}

Dass Organisationen sich beständig verändern und modernisieren müssen, ist an und für sich keine neue Entwicklung. Die Geschwindigkeit, mit der Innovation vom Markt erwartet wird, jedoch sehr wohl. Durch die Digitalisierung und Globalisierung der Märkte haben sich die Innovationszyklen stark beschleunigt. Gerade in Branchen wie im Einzelhandel oder der Automobilindustrie verändert sich der Markt fundamental. Organisationen stehen zunehmend unsicheren und diskontinuierlichen Umweltgegebenheiten gegenüber, in denen bisherige Lösungen teils zu langsam, technologisch veraltet und einfach nicht mehr ausreichend sind, um nachhaltig Wettbewerbsvorteile zu erzielen. Unternehmensbeispiele wie der Fotoausrüster Kodak oder der Quelle Versand in Deutschland werden oftmals als prominente Beispiele genannt, die infolge diskontinuierlicher (vor allem technologischer) Veränderungen der Rahmenbedingungen vom Markt verschwunden sind (vgl. Fojcik 2015, S. 1 ff).

Vor dem Hintergrund der digitalen Transformation tritt das Konzept der Ambidextrie mit dem Anspruch an, eine passende Beschreibungshilfe für die Widersprüchlich- 
keit zu liefern, mit der Unternehmen momentan zu kämpfen haben. Sowohl die Produktentwicklung sollte digital antwortfähig werden als auch interne Organisationsabläufe sich mit den Möglichkeiten neuer technologischer Bedingungen befassen. Gleichzeitig könne eine historisch gewachsene Unternehmenskultur mit ihren Prozessen, Routinen und menschlichen Verhaltenslogiken nicht von ein auf den anderen Tag verändert werden. Ambidextrie als Denkmodell, das von den veränderten Marktanforderungen in einer digitalen Welt ausgeht und der Exploitation-Tätigkeit die Exploration-Notwendigkeit als Entwicklungspol zur Seite steht, liefert Management und Führung ein nachvollziehbares Konzept, um auftretende Spannungsfelder zu beschreiben, zu verstehen und Entscheidungen daraus abzuleiten. Ambidextrie löst jedoch keine Probleme, sie ist viel mehr das Problem. Jedes Denkmodell dient letztendlich dazu, eigene Komplexität mit Hilfe von Selektion der eigenen Wahrnehmung beschreibbar und damit handhabbar zu machen. Gleichzeitig bleibt es immer nur ein möglicher Ausschnitt der organisationalen Wirklichkeit und damit anfällig für die ungeplanten Abzweigungen der Praxis.

Wie das hier diskutierte Fallbeispiel zeigt, befand sich das Unternehmen in Konfrontation mit den veränderten Marktbedingungen. Hätte man nicht begonnen, eine eigene Innovationseinheit zur Entwicklung digitaler Produktlösungen zu gründen, wäre man mittlerweile zu spät dran, um gegen die Mitbewerber bestehen zu können. Eine ambidextre Dynamik war in diesem Unternehmen mit Sicherheit zu finden, da man sowohl exploitative als auch explorative Anforderungen gleichermaßen bearbeiten musste. Was man jedoch unterschätzt hatte, war der mühsame Weg durch die kulturellen Spannungsfelder der Organisation, die mit der Paradoxie zwischen Exploitation- und Exploration-Marktausrichtung um eine Facette reicher wurden.

\subsection{Was für Ambidextrie als Managementmode spricht}

Das Zusammen- bzw. Gegeneinander-Spiel von Exploitation und Exploration scheint im Diskurs zur digitalen Transformation nicht nur für Führungsverantwortliche interessant zu sein, sondern birgt auch auf dem Beratungsmarkt Potenzial, Lösungsangebote im Konfliktfeld zwischen Verändern und Bewahren zu generieren. Viele dieser Spannungsfelder, so wie auch im Fallbeispiel beschrieben, sind dabei nicht neu, werden jedoch in der Erzählung zur Ambidextrie als neu verkauft. Organisationsimmanente Widersprüche wie Qualität vs. Effizienz, Kontinuität vs. Flexibilität, Selbstvs. Fremdsteuerung, etc. werden zu den widersprüchlichen Anforderungen von Exploitation und Exploration subsummiert, sind aber so alt wie die Erfindung der Organisation selbst (vgl. Schwarz 2010, S. 191 ff. und 231 ff. oder Simon 2007, S. 74 ff.).
Es lässt sich annehmen, dass mit der Popularität des Ambidextriebegriffs auch eine Managementmode deutlich wird, die als Legitimationskonzept für Beratungsleistung und Ratgeberliteratur in einer digitalen Welt dient (vgl. etwa Rogers 2016). Ähnlich dem Agilitätsbegriff (vgl. auch Csar 2020) gilt Ambidextrie im Sprachgebrauch der Managementberatung als Ausdruck der persönlichen Profilierung und Positionierung am Beratungsmarkt. Wie Krainz (2020) beschreibt, scheint auf der Ebene der Expert*innen ,weit wichtiger als tiefergehende theoretische Auseinandersetzungen mit solchen Konzepten (...) jedenfalls ihr Tauschund Anwendungswert am Markt zu sein. (...) Wer den neuesten Sprachjargon beherrscht, kann imponieren und signalisieren, sich auf der Höhe der Zeit zu bewegen“ (ebd. S. 493).

Ebenfalls bedenklich scheint, wenn die ambidextre Organisation als Ideal zukunftsfähiger Organisation ausgerufen wird. Arnold etwa (vgl. 2016, S. 56) setzt in seiner Matrix zum Zusammenspiel zwischen Organisationsdesign und der Rolle der Mitarbeiter*innen die ambidextre Organisation mit dem agilen Netzwerk gleich. In diesem Bild wirkt dieser Zustand als der erstrebenswerteste, da die Alternativen mit „Weisung \& Kontrolle“, „Überlastung“ oder der „Schattenorganisation“ wenig attraktiv erscheinen. Wie oftmals in den postmodernen Organisationsmodellen zu New Work (Bergman), Holacracy (Robertson) oder Teal Organisation (Laloux) herauslesbar, versteckt sich hinter der ideologisch aufgeladenen Entwicklung von Organisation eine Linearität und Dogmatik, die die Verschiedenheit der Organisationsrealitäten ausblendet und ein bevorzugtes Modell bewirbt. Ambidextrie in einem IT-Unternehmen mit 10 Mitarbeiter*innen wird anders ausgelegt werden müssen als Ambidextrie in einem Automobilkonzern. Wiederum vom Aufbau einer ambidextren Schule zu sprechen, wäre eine Verfehlung des Konzepts und Marktgeschrei aus der Berater*innenbranche schlechthin.

Der Ambidextriebegriff kann als Phänomen in Zeiten digitaler Transformationshysterie betrachtet werden, der auf einem theoretisch fundierten Konzept der Innovationsforschung beruht, der aber bei weitem nicht alle Widerspruchsdynamiken und organisationalen Paradoxien miteinschließt. Er bietet einen hilfreichen Blickwinkel, sofern er sich auf die Frage bezieht, wie Organisationen auftretende Innovationsherausforderungen bewältigen und aus ihren angestammten geschäftspolitischen Pfaden aussteigen lernen können (vgl. Schumacher und Wimmer 2018, S. $11 \mathrm{ff}$ ). Er bleibt Ausdruck oberflächlicher Berater*innenund Managementsprache, sofern in ihm der heilige Gral zur Lösung organisationaler Konfliktfelder in einer digitalen Welt gesehen und er als ein solcher kommuniziert wird. 


\section{Zusammenfassung und Ausblick}

Der Text stellt einen Beitrag zur Reflexion des Ambidextriebegriffs dar. Zu Beginn wurde erläutert, dass es sich dabei um ein Konzept der 1990er-Jahre handelt, das die Spannungspole Exploitation und Exploration in der Entwicklung von Innovation und organisationalem Lernen aufgreift. In Folge wurde an Hand eines Fallbeispiels versucht Konfliktdynamiken in der Entwicklung ambidextrer Strukturen in der Praxis zu beleuchten. Im Kontext von Veränderungsprozessen in Organisationen erscheinen diese als nicht sonderlich neu. Widersprüche und organisationale Paradoxien müssen auch abseits des Ambidextriekonzepts permanent gemanagt und balanciert werden. Neu ist, dass in der Ausrichtung auf veränderte Marktdynamiken (Erfordernis zur Entwicklung digitaler Produkte) dem Spannungsfeld zwischen Exploitation und Exploration in der Wertschöpfung besondere Bedeutung gegeben wird. Hier kann das Ambidextriemodell nützliche Beschreibungselemente liefern, worauf sich Organisation und Führung in der Reaktion auf die widersprüchlichen Erwartungen vom Markt einstellen müssen. Für das Management stellt der Ambidextriebegriff somit ein willkommenes Instrument dar, Widersprüchlichkeit und Zerrissenheit in der eigenen strategischen Ausrichtung zu benennen und bewusster damit zu agieren.

In der Managementliteratur findet man genügend Handlungsleitlinien und Empfehlungen, wie der Aufbau einer ambidextren Organisation gelingen kann bzw. was aus Führungsperspektive zu beachten ist. Tushman und O'Reilly (2016) definieren nach einer Metaanalyse zur Steuerung von Unternehmen im disruptiven Bereich verschiedene Kriterien zur Fähigkeit organisationaler Ambidextrie. Neben den regelmäßigen Anpassungsschleifen zur Entscheidungsfindung in den unterschiedlichen Organisationseinheiten stehen bewusstes Führen, ein offenes Austragen von Spannungen und Konflikten im Führungsteam sowie eine emotional ansprechende strategische Absicht und Vision des Topmanagements zur Verbindung beider Welten an erster Stelle (vgl. ebd. S. 174-175).

Szlang und Bruch (2020) unterstützen in ihrer Ausführung die besonderen Anforderungen an Führung. Diese muss auf der einen Seite stabilisierendes, effizienzsicherndes Verhalten an den Tag legen, das Zieleinhaltung kontrolliert und auf Routinen setzt. Auf der anderen Seite muss Führung dazu beitragen, dass Mitarbeiter*innen beginnen eigene Ideen $\mathrm{zu}$ entwickeln, Routinen $\mathrm{zu}$ verlassen und eigene Entscheidungen zu treffen (vgl. ebd. S. 194). Die konsequente Arbeit und Reflexion der Führungshaltung sowie die bewusste Auseinandersetzung mit den widersprüchlichen Erwartungen und Verhaltensaufforderungen an die Führungskraft stellen die Voraussetzung für das Gelingen ambidextrer Unternehmensentwicklung dar.
Schumacher und Wimmer (2018, S. 18) weisen wiederum darauf hin, dass der Fokus auf die Gestaltung eines passenden Organisationsdesigns gelegt werden muss. Organisationen müssen strukturelle Antworten finden, um gegenüber den höchst volatilen und widersprüchlichen externen Anforderungen von Effizienz und Innovation antwortfähig zu bleiben. Das Verstehen der eigenen organisationalen Binnendifferenzierung sowie die sorgfältige Pflege einer ausgeprägten Innovationskultur können dabei helfen.

In der Zusammenschau der Leitlinien zum Aufbau organisationaler Ambidextrie wird deutlich, dass die Diskussion über die Entwicklung von Führungsgrundsätzen hinausreichen muss. Organisationale Ambidextrie stellt mit der Anforderung, zwei diametral zueinanderstehende Markterwartungen zu steuern, die bestehenden Organisationsstrukturen von Grund auf in Frage. Wer also Ambidextrie will, muss lernen Konflikte innerhalb der Teams und Abteilungen zu managen und passende Rahmenbedingungen innerhalb der Organisation dafür zu schaffen. Sowohl die Förderung gruppendynamischen Know Hows in der Führungskräfteentwicklung (vgl. Csar 2018) als auch die Etablierung einer widerspruchsfähigen Unternehmenskultur durch den regelmäßigen Austausch und das Aushandeln der Konfliktlinien auf Teamebene, sind notwendige Begleitmaßnahmen zur gesamten Anpassung der Organisation mit ihren Rollen, Strukturen und Prozessen.

Letztendlich bleibt zu überprüfen, ob Ambidextrie die Antwort auf die Umweltveränderungen eines Unternehmens ist. Denn sofern es sich dabei nicht um die Ungewissheiten der digitalen Transformation und deren Notwendigkeit handelt, dem bestehenden Geschäft der Gegenwart die Innovation der Zukunft zur Seite zu stellen, bleibt die Nutzung des Ambidextriebegriffs wohl mehr eine angesagte Umschreibung aktueller Konfliktdynamiken heutiger Organisationen.

Funding Open access funding provided by FH Salzburg - University of Applied Sciences.

Open Access Dieser Artikel wird unter der Creative Commons Namensnennung 4.0 International Lizenz veröffentlicht, welche die Nutzung, Vervielfältigung, Bearbeitung, Verbreitung und Wiedergabe in jeglichem Medium und Format erlaubt, sofern Sie den/die ursprünglichen Autor(en) und die Quelle ordnungsgemäß nennen, einen Link zur Creative Commons Lizenz beifügen und angeben, ob Änderungen vorgenommen wurden.

Die in diesem Artikel enthaltenen Bilder und sonstiges Drittmaterial unterliegen ebenfalls der genannten Creative Commons Lizenz, sofern sich aus der Abbildungslegende nichts anderes ergibt. Sofern das betreffende Material nicht unter der genannten Creative Commons Lizenz steht und die betreffende Handlung nicht nach gesetzlichen Vorschriften erlaubt ist, ist für die oben aufgeführten Weiterverwendungen des Materials die Einwilligung des jeweiligen Rechteinhabers einzuholen.

Weitere Details zur Lizenz entnehmen Sie bitte der Lizenzinformation auf http://creativecommons.org/licenses/by/4.0/deed.de. 


\section{Literatur}

Arnold, H. (2016). Wir sind Chef. Freiburg: Haufe.

Birkinshaw, J., \& Gupta, A. K. (2013). Clarifying the distinctive contribution of ambidexterity to the field of organization studies. Academy of Management Perspectives, 27(4), 287-298.

Csar, M. (2018). Organisationen im Umbruch: Zur Aktualität gruppendynamischen Lernens in der Ausbildung von MitarbeiterInnen der Zukunft. In Tagungsband, FFH Konferenz 2018.

Csar, M. (2020). Agilität als Ziel von Veränderungsprozessen. Über Sinn und Unsinn in der Einführung von Agilität in Organisationen. Gruppe. Interaktion. Organisation. Zeitschrift für Angewandte Organisationspsychologie (GIO), 51(4), 391-401.

Duncan, R. (1976). The ambidextrous organization: designing dual structures for innovation. In R. H. Killman, L. R. Pondy \& D. Sleven (Hrsg.), The management of organization (S. 167-188). New York: North Holland.

Duwe, J. (2018). Beidhändige Führung: Wie Sie als Führungskraft in großen Organisationen Innovationssprünge ermöglichen. Berlin, Heidelberg, New York: Springer.

Exner, A., Exner, H., \& Mitterer, G. (2020). Führen in der Zerrissenheit. Duale Steuerung in wachsenden Familienunternehmen. Organisationsentwicklung, 2020(4), 64-69.

Fojcik, T. (2015). Ambidextrie und Unternehmenserfolg bei einem diskontinuierlichen Wandel. Wiesbaden: Springer.

Gergs, H. J., \& Lakeit, A. (2020). Agilität braucht Stabilität. Mit Ambidextrie Neues schaffen und Bewährtes bewahren. Stuttgart: Schäffer-Poeschel.

Gibson, C.B., \& Birkinshaw, J. (2004). The antecedents, consequences, and mediating role of organizational ambidexterity. Academy of Management Journal, 47(2), 209-226.

Glasl, F. (2013). Konfliktmanagement - Ein Handbuch für Führungskräfte, Beraterinnen und Berater (11. Aufl.). Bern: Verlag Freies Geistesleben.

Hernstein GmbH (2020). Agilität und Hierarchie: Können Führungskräfte beidhändig führen? In: Hernstein Management Report 1/2020. https://www.hernstein.at/fileadmin/user_upload/HMR/ HMR_1_2020_Ambidextrie.pdf. Zugegriffen: 20. Okt. 2020.

Krainz, U. (2020). Jenseits des Marktprinzips. Über den politischen Sinn gruppendynamischen Lernens. Gruppe. Interaktion. Organisation. Zeitschrift für Angewandte Organisationspsychologie (GIO), 51(4), 489-498.

Kuster, J., et al. (2018). Handbuch Projektmanagement. Agil - Klassisch - Hybrid (4. Aufl.). Wiesbaden: Gabler.

Lavie, D., et al. (2010). Exploration and Exploitation within and across organizations. Academy of Management Annals, 4(1), 109-155.

Levinthal, D. A., \& March, J. G. (1993). The myopia of learning. Strategic Management Journal, 14(S2), 95-112.

March, J.G. (1991). Exploration and exploitation in organizational learning. Organization Science, 2(1), 71-87.

Rogers, D.L. (2016). The digital transformation playbook. Rethink your business for the digital age. : Columbia University Press.
Schneeberger, S. J., \& Habegger, A. (2020). Ambidextrie - der organisationale Drahtseilakt. In J. Schellinger, K. O. Tokarski \& I. Kissling-Näf (Hrsg.), Digitale Transformation und Unternehmensführung. Trends und Perspektiven für die Praxis (S. 105-144). Wiesbaden: Gabler.

Schumacher, T. (2020). Ambidextrie gestern und heute. Ein Interview mit Michael Tushman. Organisationsentwicklung, 2020(4), 4-9.

Schumacher, T., \& Wimmer, R. (2018). Gleichzeitig optimieren und neu erfinden? Organisationsentwicklung, 2018(1), 10-17.

Schwarz, G. (2010). Konfliktmanagement. Konflikte erkennen, analysieren, lösen (8. Aufl.). Wiesbaden: Gabler.

Simon, F. B. (2007). Paradoxiemanagement - Genie und Wahnsinn der Organisation. Revue für postheroisches Management, 1, 68-87.

Szlang, J., \& Bruch, H. (2020). Ein ambidextres Führungsklima - Erfolgsfaktoren in der neuen Arbeitswelt. Gruppe. Interaktion. Organisation. Zeitschrift für Angewandte Organisationspsychologie (GIO), 51(2), 187-197.

Timinger, H. (2017). Modernes Projektmanagement: Mit traditionellem, agilem und hybridem Vorgehen zum Erfolg. Weinheim: Wiley-VCH.

Tushman, M.L., \& O’Reilly, C.A. (1996). Ambidextrous organizations: managing evolutionary and revolutionary change. California Management Review, 38(4), 8-30.

Tushman, M.L., \& O'Reilly, C. A. (2004). The ambidextrous organization. Harvard Business Review, 82(4), 74-81.

Tushman, M.L., \& O’Reilly, C. A. (2013). Organizational ambidexterity: past, present and future. Academy of Management Perspectives, 27(4), 324-338.

Tushman, M.L., \& O'Reilly, C. A. (2016). Lead and disrupt: how to solve the innovator's dilemma. Stanford: University Press.

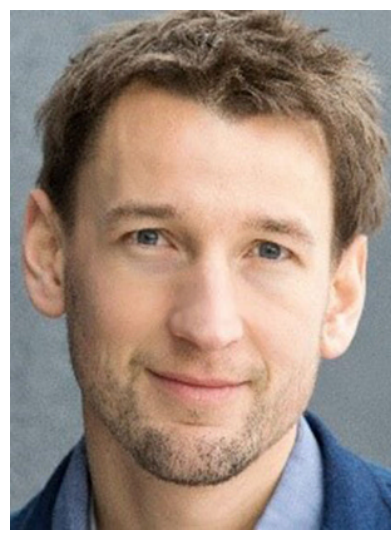

Matthias Csar arbeitet als Berater und Organisationsentwickler mit Schwerpunkt Führung, Gruppenund Organisationsdynamik. An der Fachhochschule Salzburg lehrt er als Fachbereichsleiter für Sozial\& Kommunikationskompetenz im Studiengang Informationstechnik. Sowohl in der Lehre als auch im Bereich Training und Beratung beschäftigt er sich mit Trends zu neuen Organisationsmodellen und deren Auswirkung auf das Thema Führung und die Zusammenarbeit in Teams. Matthias Csar ist Mitglied der ÖGGO (Österr. Gesellschaft für Gruppedynamik \& Organisationsberatung). 